Supporting Information

\title{
Epigenetic Target Fishing with Accurate Machine Learning Models
}

Norberto Sánchez-Cruz and José L. Medina-Franco

DIFACQUIM Research Group, Department of Pharmacy, School of Chemistry, Universidad Nacional Autónoma de México, Mexico City 04510, Mexico

KEYWORDS: Cheminformatics; Chemogenomics; Drug Discovery; Epigenetics; Epi-Informatics; Machine learning; Structure-activity relationships.

Contents Page

Table S1. Target-associated compound datasets included in this work.

Table S2. Models with no significant difference of performance.

Table S3. Single Target Performance.

Table S4. Distance-to-model quartiles.

Figure S1. Performance comparison of single target binary classifiers.

Figure S2. Compounds overlap between samples employed in the Multi-Target Validation.

Table S5. External compounds employed for retrospective target prediction.

Table S6. Targets predicted by the consensus model for compound 17 .

Table S7. Targets predicted by HitPickV2 for compound 17.

Table S8. Targets predicted by PPB for compound 17.

Table S9. Targets predicted by SEA for compound 17.

Table S10. Top 45 targets predicted by SwissTargetPrediction for compound 17 .

Table S11. Targets predicted by the consensus model for compound 43a.

Table S12. Targets predicted by HitPickV2 for compound 43a.

Table S13. Targets predicted by PPB for compound 43a.

Table S14. Targets predicted by SEA for compound 43a.

Table S15. Top 45 targets predicted by SwissTargetPrediction for compound 43a. 
Table S1. Target-associated compound datasets included in this work.

\begin{tabular}{|c|c|c|c|c|c|c|c|}
\hline Target & Active compounds & Inactive compounds & MODI_MACCS & MODI_Morgan & MODI_RDK & Level1 & Level2 \\
\hline APEX1 & 39 & 99 & 0.862 & 0.847 & 0.870 & Other & Cofactor \\
\hline ATM & 31 & 51 & 0.757 & 0.796 & 0.793 & Writer & KIN \\
\hline AURKA & 2171 & 497 & 0.783 & 0.806 & 0.808 & Writer & KIN \\
\hline AURKB & 1440 & 328 & 0.793 & 0.823 & 0.819 & Writer & KIN \\
\hline BRD2 & 216 & 31 & 0.790 & 0.823 & 0.804 & Reader & BRD \\
\hline BRD4 & 1559 & 322 & 0.804 & 0.815 & 0.828 & Reader & BRD \\
\hline BRPF1 & 84 & 50 & 0.749 & 0.776 & 0.757 & Reader & BRD \\
\hline CARM1 & 63 & 34 & 0.769 & 0.866 & 0.812 & Writer & HMT \\
\hline CDK1 & 775 & 504 & 0.847 & 0.879 & 0.867 & Writer & KIN \\
\hline CDK2 & 1312 & 722 & 0.804 & 0.835 & 0.835 & Writer & KIN \\
\hline CDK5 & 142 & 205 & 0.821 & 0.846 & 0.836 & Writer & KIN \\
\hline CDK7 & 106 & 106 & 0.670 & 0.679 & 0.726 & Writer & KIN \\
\hline CHEK1 & 1379 & 403 & 0.819 & 0.835 & 0.830 & Writer & KIN \\
\hline CHUK & 266 & 173 & 0.770 & 0.806 & 0.817 & Writer & KIN \\
\hline CREBBP & 254 & 103 & 0.778 & 0.780 & 0.819 & Writer & HAT \\
\hline DAPK3 & 52 & 67 & 0.716 & 0.673 & 0.683 & Writer & KIN \\
\hline DNMT1 & 89 & 62 & 0.808 & 0.858 & 0.847 & Writer & DNMT1 \\
\hline DOT1L & 56 & 45 & 0.936 & 0.907 & 0.898 & Writer & HMT \\
\hline EHMT2 & 203 & 63 & 0.751 & 0.848 & 0.814 & Writer & HMT \\
\hline EP300 & 62 & 38 & 0.730 & 0.661 & 0.642 & Writer & HAT \\
\hline $\mathrm{EZH} 2$ & 265 & 39 & 0.765 & 0.818 & 0.805 & Writer & HMT \\
\hline HDAC1 & 2605 & 532 & 0.810 & 0.815 & 0.795 & Eraser & HDAC \\
\hline HDAC10 & 196 & 72 & 0.827 & 0.838 & 0.855 & Eraser & HDAC \\
\hline HDAC11 & 134 & 76 & 0.795 & 0.841 & 0.807 & Eraser & HDAC \\
\hline HDAC2 & 856 & 198 & 0.817 & 0.791 & 0.806 & Eraser & HDAC \\
\hline HDAC3 & 973 & 239 & 0.815 & 0.815 & 0.810 & Eraser & HDAC \\
\hline HDAC4 & 188 & 246 & 0.833 & 0.841 & 0.851 & Eraser & HDAC \\
\hline HDAC5 & 99 & 159 & 0.840 & 0.849 & 0.864 & Eraser & HDAC \\
\hline HDAC6 & 1632 & 389 & 0.896 & 0.858 & 0.818 & Eraser & HDAC \\
\hline HDAC7 & 101 & 155 & 0.818 & 0.810 & 0.790 & Eraser & HDAC \\
\hline HDAC8 & 855 & 374 & 0.878 & 0.872 & 0.865 & Eraser & HDAC \\
\hline HDAC9 & 103 & 122 & 0.812 & 0.862 & 0.848 & Eraser & HDAC \\
\hline JAK2 & 4529 & 372 & 0.816 & 0.850 & 0.855 & Writer & KIN \\
\hline KAT2B & 53 & 59 & 0.717 & 0.707 & 0.777 & Writer & HAT \\
\hline KDM1A & 330 & 117 & 0.853 & 0.852 & 0.880 & Eraser & HDM \\
\hline KDM4A & 31 & 57 & 0.660 & 0.798 & 0.832 & Eraser & HDM \\
\hline KDM4C & 98 & 101 & 0.864 & 0.915 & 0.905 & Eraser & HDM \\
\hline KDM4E & 34 & 39 & 0.778 & 0.826 & 0.866 & Eraser & HDM \\
\hline KDM5A & 49 & 112 & 0.903 & 0.959 & 0.893 & Eraser & HDM \\
\hline KDM6B & 44 & 58 & 0.780 & 0.814 & 0.774 & Eraser & HDM \\
\hline L3MBTL1 & 50 & 62 & 0.791 & 0.839 & 0.815 & Reader & L3MBTL1 \\
\hline PARG & 87 & 48 & 0.835 & 0.861 & 0.818 & Other & $\mathrm{CHR}$ \\
\hline PARP1 & 2560 & 230 & 0.788 & 0.808 & 0.827 & Other & $\mathrm{CHR}$ \\
\hline PKN1 & 36 & 82 & 0.694 & 0.746 & 0.737 & Writer & KIN \\
\hline
\end{tabular}




\begin{tabular}{ccccccccc} 
PRKAA1 & 36 & 63 & 0.685 & 0.740 & 0.734 & Writer & KIN \\
PRKCB & 527 & 115 & 0.827 & 0.822 & 0.815 & Writer & HMT \\
PRKCD & 506 & 142 & 0.865 & 0.903 & 0.872 & Writer & KIN \\
PRKDC & 799 & 94 & 0.716 & 0.643 & 0.654 & Writer & KIN \\
PRMT3 & 60 & 41 & 0.750 & 0.746 & 0.774 & Writer & HMT \\
RPS6KA5 & 74 & 101 & 0.835 & 0.856 & 0.886 & Writer & KIN \\
SIRT1 & 140 & 274 & 0.849 & 0.831 & 0.829 & Eraser & HDAC/HDM \\
SIRT2 & 212 & 321 & 0.771 & 0.776 & 0.785 & Eraser & HDAC/HDM \\
SIRT3 & 39 & 63 & 0.872 & 0.857 & 0.877 & Eraser & HDAC \\
TOP2A & 81 & 268 & 0.809 & 0.792 & 0.832 & Other & CHR \\
USP7 & 69 & 56 & 0.793 & 0.804 & 0.684 & Eraser & USP7 \\
\hline
\end{tabular}


Table S2. Models with no significant difference of performance.

\begin{tabular}{cl} 
Target & Models with no significant difference of performance compared to the best model \\
\hline \multirow{2}{*}{ APEX1 } & Morgan:::FFNN, Morgan::GBT, RDK::k-NN, MACCS::GBT, Morgan:::SVM, RDK::FFNN, RDK::SVM, MACCS::RF, \\
& RDK::GBT, MACCS::SVM, MACCS::k-NN, MACCS::FFNN, RDK::RF \\
ATM & RDK::GBT, MACCS::SVM, RDK::SVM, Morgan::FFNN, RDK::RF, Morgan::k-NN, RDK:::FFNN, Morgan::SVM, \\
& Morgan::GBT, RDK::k-NN, Morgan::RF, MACCS::k-NN
\end{tabular}
AURKA RDK::SVM, RDK::GBT, RDK::FFNN, MACCS::SVM
AURKB RDK::SVM, RDK::GBT, RDK::FFNN, Morgan::k-NN, Morgan::FFNN, RDK::k-NN, Morgan::SVM
BRD2 Morgan::SVM, RDK::GBT, Morgan::k-NN, RDK::SVM, Morgan::GBT, MACCS::SVM, MACCS::k-NN, Morgan::FFNN,

BRD4 RDK::GBT, Morgan::SVM, RDK::SVM, RDK::RF, MACCS::GBT, RDK::FFNN, MACCS::SVM
BRPF1 MACCS::FFNN, MACCS::SVM, Morgan::SVM, Morgan::FFNN, Morgan::GBT, Morgan::RF, MACCS::GBT, MACCS::RF, RDK::SVM, Morgan::k-NN, RDK::FFNN, RDK::GBT, RDK::k-NN, MACCS::k-NN CARM1 RNK::FFNN, Morgan::FFNN, Morgan::SVM, Morgan::GBT, Morgan::k-NN, RDK::SVM, Morgan::RF, RDK::GBT, RDK::k-

CDK1 Morgan::k-NN, RDK::GBT, Morgan::SVM, Morgan::GBT, RDK::SVM, RDK::RF

CDK2 RDK::GBT, RDK::SVM, RDK::RF, RDK::k-NN

CDK5 Morgan::k-NN, Morgan::FFNN, Morgan::SVM, RDK::FFNN, RDK::GBT, RDK::SVM, Morgan::RF, Morgan::GBT, RDK::RF, RDK::k-NN, MACCS::k-NN, MACCS::GBT, MACCS::RF, MACCS::SVM

CDK7 Morgan::GBT, RDK::FFNN, RDK::k-NN, MACCS::SVM, RDK::RF, RDK::SVM, Morgan::SVM, RDK::GBT, MACCS::FFNN, Morgan::FFNN, MACCS::GBT, MACCS::k-NN, Morgan::RF, MACCS::RF, Morgan::k-NN

CHEK1 RDK::GBT, RDK::FFNN, Morgan::SVM, RDK::SVM, Morgan::FFNN

CHUK Morgan::GBT, RDK::GBT, Morgan::SVM, Morgan::RF, RDK::RF, RDK::SVM, RDK::FFNN

CREBBP RDK::GBT, RDK::SVM, Morgan::SVM

DAPK3 Morgan::GBT, MACCS::k-NN, MACCS::GBT, RDK::GBT, RDK::FFNN, Morgan::SVM, MACCS::RF, MACCS::FFNN, MACCS::SVM, Morgan::RF, Morgan::k-NN, RDK::SVM, RDK::RF, Morgan::FFNN, RDK::k-NN

DNMT1 Morgan::SVM, RDK::SVM, Morgan::FFNN, Morgan::RF, RDK::RF, MACCS::SVM, RDK::FFNN, Morgan::GBT, RDK::GBT, MACCS::GBT, MACCS::RF, MACCS::FFNN, MACCS::k-NN

DOT1L Morgan::SVM, Morgan::RF, MACCS::SVM, Morgan::FFNN, MACCS::GBT, MACCS::RF, RDK::GBT, Morgan::k-NN, MACCS::k-NN, RDK::k-NN

EHMT2 RDK::GBT, RDK::FFNN, Morgan::FFNN, RDK::SVM, Morgan::SVM, Morgan::GBT, RDK::RF, Morgan::RF

EP300 MACCS::RF, MACCS::k-NN, Morgan::SVM, MACCS::SVM, MACCS::FFNN, RDK::SVM, MACCS::GBT, Morgan::FFNN, RDK::FFNN, RDK::RF, RDK::GBT, RDK::k-NN

EZH2 Morgan::GBT, Morgan::SVM, Morgan::k-NN, RDK::SVM, Morgan::RF, RDK::FFNN, RDK::GBT, Morgan::FFNN, RDK::kNN, RDK::RF, MACCS::FFNN, MACCS::GBT, MACCS::SVM

HDAC1 Morgan::FFNN, Morgan::SVM, RDK::SVM, Morgan::k-NN, MACCS::GBT, RDK::FFNN

HDAC10 RDK::k-NN, MACCS::GBT, Morgan::SVM, MACCS::SVM, Morgan::FFNN, Morgan::k-NN, RDK::GBT, RDK::SVM, Morgan::GBT, RDK::FFNN, MACCS::RF, Morgan::RF, MACCS::FFNN

HDAC11 RDK::GBT, Morgan::GBT, RDK::FFNN, RDK::SVM, Morgan::k-NN, Morgan::SVM, MACCS::SVM, Morgan::FFNN, MACCS::GBT, RDK::RF, MACCS::RF

HDAC2 Morgan::SVM, RDK::GBT, MACCS::GBT, MACCS::SVM

HDAC3 Morgan::SVM, Morgan::GBT

HDAC4 RDK::k-NN, Morgan::GBT, RDK::SVM, Morgan::SVM, RDK::GBT, Morgan::RF, Morgan::FFNN, RDK::FFNN, RDK::RF, MACCS::k-NN, Morgan::k-NN, MACCS::FFNN, MACCS::GBT, MACCS::SVM, MACCS::RF

HDAC5 Morgan::FFNN, RDK::GBT, Morgan::SVM, RDK::FFNN, RDK::SVM, Morgan::GBT, RDK::RF, RDK::k-NN, MACCS::GBT, Morgan::RF, MACCS::SVM, Morgan::k-NN, MACCS::k-NN, MACCS::FFNN

HDAC6 Morgan::SVM, Morgan::GBT, Morgan::RF, MACCS::RF, MACCS::GBT 
RDK::FFNN, Morgan::FFNN, Morgan::SVM, MACCS::k-NN, RDK::GBT, Morgan::k-NN, MACCS::GBT, RDK::k-NN, MACCS::RF, Morgan::RF, MACCS::SVM, RDK::SVM, RDK::RF, Morgan::GBT, MACCS::FFNN

HDAC8 Morgan::SVM, Morgan::GBT, Morgan::RF, MACCS::GBT, MACCS::FFNN

HDAC9 RDK::GBT, RDK::RF, Morgan::k-NN, Morgan::RF, RDK::SVM, RDK::FFNN, Morgan::GBT, RDK::k-NN, Morgan::FFNN, MACCS::GBT, MACCS::k-NN, MACCS::RF

JAK2 Morgan::FFNN, RDK::k-NN, RDK::FFNN, RDK::SVM, Morgan::k-NN

KAT2B

RDK::GBT, Morgan::GBT, RDK::k-NN, RDK::RF, RDK::FFNN, RDK::SVM, Morgan::SVM, Morgan::RF, MACCS::GBT, MACCS::k-NN, Morgan::k-NN, Morgan::FFNN, MACCS::RF, MACCS::SVM, MACCS::FFNN

KDM1A RDK::GBT, RDK::RF, MACCS::SVM, RDK::SVM, MACCS::GBT, RDK::FFNN, MACCS::RF, RDK::k-NN, MACCS::FFNN, Morgan::SVM, Morgan::GBT, Morgan::RF, Morgan::FFNN, Morgan::k-NN

KDM4A RDK::GBT, RDK::FFNN, RDK::SVM, RDK::RF, Morgan::FFNN

KDM4C Morgan::SVM, RDK::SVM, Morgan::RF, RDK::GBT, MACCS::RF, MACCS::GBT, MACCS::SVM, RDK::RF, Morgan::GBT, RDK::FFNN, RDK::k-NN, Morgan::FFNN

KDM4E MACCS::GBT, Morgan::FFNN, MACCS::FFNN, MACCS::SVM, RDK::k-NN, RDK::FFNN, MACCS::RF, Morgan::RF, RDK::SVM, RDK::RF, RDK::GBT, Morgan::k-NN, Morgan::GBT, Morgan::SVM

KDM5A Morgan::k-NN, MACCS::GBT, MACCS::RF, MACCS::FFNN, MACCS::SVM, RDK::GBT, Morgan::SVM, Morgan::FFNN, Morgan::GBT, RDK::SVM, Morgan::RF, RDK::FFNN, RDK::RF

KDM6B Morgan::GBT, MACCS::k-NN, RDK::SVM, RDK::k-NN, Morgan::k-NN, MACCS::FFNN, Morgan::FFNN, RDK::GBT, MACCS::GBT, Morgan::SVM, MACCS::SVM, Morgan::RF, RDK::RF, RDK::FFNN

L3MBTL1 Morgan::k-NN, Morgan::RF, Morgan::FFNN, Morgan::SVM, RDK::GBT, Morgan::GBT, RDK::RF, RDK::FFNN, MACCS::SVM, MACCS::FFNN, MACCS::GBT, RDK::SVM, RDK::k-NN, MACCS::RF

PARG Morgan::FFNN, RDK::RF, MACCS::SVM, Morgan::SVM, Morgan::GBT, Morgan::RF, RDK::GBT, RDK::FFNN, Morgan::kNN, RDK::SVM, MACCS::k-NN

PARP1 RDK::SVM, RDK::FFNN, Morgan::FFNN, Morgan::SVM, RDK::GBT, Morgan::GBT, RDK::k-NN, Morgan::k-NN, Morgan::RF, MACCS::FFNN

PKN1 Morgan::SVM, RDK::FFNN, Morgan::FFNN, RDK::GBT, MACCS::SVM, RDK::k-NN, MACCS::FFNN, MACCS::GBT, RDK::SVM, Morgan::k-NN, MACCS::k-NN, Morgan::GBT, MACCS::RF

PRKAA1 Morgan::GBT, RDK::SVM, RDK::GBT, RDK::FFNN, Morgan::FFNN, Morgan::SVM, MACCS::GBT, Morgan::k-NN, Morgan::RF, RDK::RF, MACCS::SVM, RDK::k-NN, MACCS::RF, MACCS::FFNN

PRKCB RDK::GBT, RDK::RF, Morgan::SVM, Morgan::FFNN, RDK::SVM, Morgan::RF, Morgan::k-NN, MACCS::FFNN

PRKCD RDK::GBT, RDK::SVM, RDK::RF, Morgan::SVM, Morgan::FFNN, RDK::FFNN

PRKDC Morgan::SVM, RDK::GBT, RDK::FFNN, RDK::k-NN, Morgan::FFNN, MACCS::k-NN, MACCS::FFNN, Morgan::k-NN, RDK::SVM, MACCS::RF, MACCS::GBT, MACCS::SVM

PRMT3 RDK::GBT, MACCS::FFNN, MACCS::GBT, RDK::SVM, RDK::FFNN, MACCS::SVM, RDK::RF, Morgan::SVM, Morgan::RF, Morgan::k-NN, Morgan::GBT, RDK::k-NN

RPS6KA5 Morgan::FFNN, MACCS::GBT, RDK::GBT, RDK::FFNN, RDK::SVM, RDK::RF, Morgan::SVM, Morgan::RF, MACCS::FFNN, MACCS::SVM, Morgan::GBT, Morgan::k-NN, RDK::k-NN, MACCS::RF, MACCS::k-NN

SIRT1 RDK::SVM, Morgan::GBT, RDK::GBT, MACCS::k-NN, Morgan::k-NN, Morgan::RF, Morgan::FFNN, MACCS::RF, MACCS::GBT, RDK::FFNN, MACCS::SVM, RDK::k-NN, MACCS::FFNN

SIRT2 RDK::RF, RDK::GBT, Morgan::k-NN, Morgan::GBT, Morgan::SVM, RDK::k-NN, RDK::SVM, MACCS::GBT, Morgan::FFNN, MACCS::RF, MACCS::FFNN, RDK::FFNN

SIRT3 Morgan::k-NN, Morgan::SVM, Morgan::GBT, Morgan::FFNN, RDK::GBT, MACCS::SVM, RDK::k-NN, RDK::SVM, MACCS::k-NN, RDK::FFNN, Morgan::RF, RDK::RF, MACCS::GBT, MACCS::RF, MACCS::FFNN

TOP2A RDK::SVM, RDK::k-NN, RDK::FFNN, RDK::GBT, Morgan::SVM, Morgan::GBT, Morgan::FFNN, Morgan::k-NN, MACCS::k-NN

USP7

Morgan::FFNN, Morgan::GBT, Morgan::k-NN, MACCS::GBT, MACCS::SVM, Morgan::RF, Morgan::SVM, MACCS::FFNN, RDK::GBT, MACCS::RF, MACCS::k-NN, RDK::SVM

The model with the highest mean of BA for each target is shown in bold. The remaining models are sorted by decreasing mean BA. 
Table S3. Single Target Performance.

\begin{tabular}{ccccc}
\hline Model & TPR & PPV & TNR & NPV \\
\hline Consensus & $0.819 \pm 0.145$ & $0.896 \pm 0.070$ & $0.851 \pm 0.096$ & $0.787 \pm 0.097$ \\
Morgan::SVM & $0.856 \pm 0.130$ & $0.876 \pm 0.075$ & $0.805 \pm 0.119$ & $0.824 \pm 0.087$ \\
RDK::SVM & $0.864 \pm 0.120$ & $0.865 \pm 0.080$ & $0.790 \pm 0.118$ & $0.822 \pm 0.086$ \\
RDK::GBT & $0.856 \pm 0.136$ & $0.870 \pm 0.077$ & $0.793 \pm 0.124$ & $0.822 \pm 0.080$ \\
RDK::FFNN & $0.859 \pm 0.111$ & $0.862 \pm 0.081$ & $0.785 \pm 0.115$ & $0.813 \pm 0.081$ \\
Morgan::FFNN & $0.857 \pm 0.111$ & $0.856 \pm 0.092$ & $0.782 \pm 0.116$ & $0.808 \pm 0.086$ \\
Morgan::k-NN & $0.871 \pm 0.121$ & $0.850 \pm 0.090$ & $0.762 \pm 0.121$ & $0.828 \pm 0.093$ \\
RDK::RF & $0.856 \pm 0.149$ & $0.870 \pm 0.071$ & $0.777 \pm 0.145$ & $0.835 \pm 0.073$ \\
Morgan::GBT & $0.852 \pm 0.145$ & $0.869 \pm 0.078$ & $0.779 \pm 0.140$ & $0.824 \pm 0.089$ \\
RDK::k-NN & $0.873 \pm 0.104$ & $0.840 \pm 0.096$ & $0.754 \pm 0.115$ & $0.816 \pm 0.095$ \\
Morgan::RF & $0.853 \pm 0.158$ & $0.871 \pm 0.072$ & $0.770 \pm 0.162$ & $0.841 \pm 0.076$ \\
MACCS::SVM & $0.851 \pm 0.136$ & $0.846 \pm 0.101$ & $0.763+0.126$ & $0.805 \pm 0.104$ \\
MACCS::GBT & $0.846 \pm 0.137$ & $0.846 \pm 0.096$ & $0.765 \pm 0.126$ & $0.801 \pm 0.089$ \\
MACCS::RF & $0.849 \pm 0.140$ & $0.846 \pm 0.088$ & $0.751 \pm 0.144$ & $0.810 \pm 0.082$ \\
MACCS::k-NN & $0.856 \pm 0.124$ & $0.826 \pm 0.101$ & $0.727 \pm 0.125$ & $0.793 \pm 0.109$ \\
MACCS::FFNN & $0.835 \pm 0.121$ & $0.824 \pm 0.111$ & $0.735 \pm 0.122$ & $0.766 \pm 0.103$
\end{tabular}

Mean and standard deviation (mean \pm SD) of TPR, PPV, TNR and NPV for 55 single target binary classifiers built on 15 fingerprint::algorithm combinations and a consensus model.

Table S4. Distance-to-model quartiles.

\begin{tabular}{cc}
\hline Quartile & Mean Jaccard distance \\
\hline Q1 & {$[0.0,0.820]$} \\
Q2 & $(0.820,0.844]$ \\
Q3 & $(0.844,0.865]$ \\
Q4 & $(0.865,1.0]$ \\
\hline
\end{tabular}

The minimum and maximum values of Jaccard distance were 0.493 and 0.977 , ranges for Q1 and Q4 were extended to cover all possible values. 
(a)

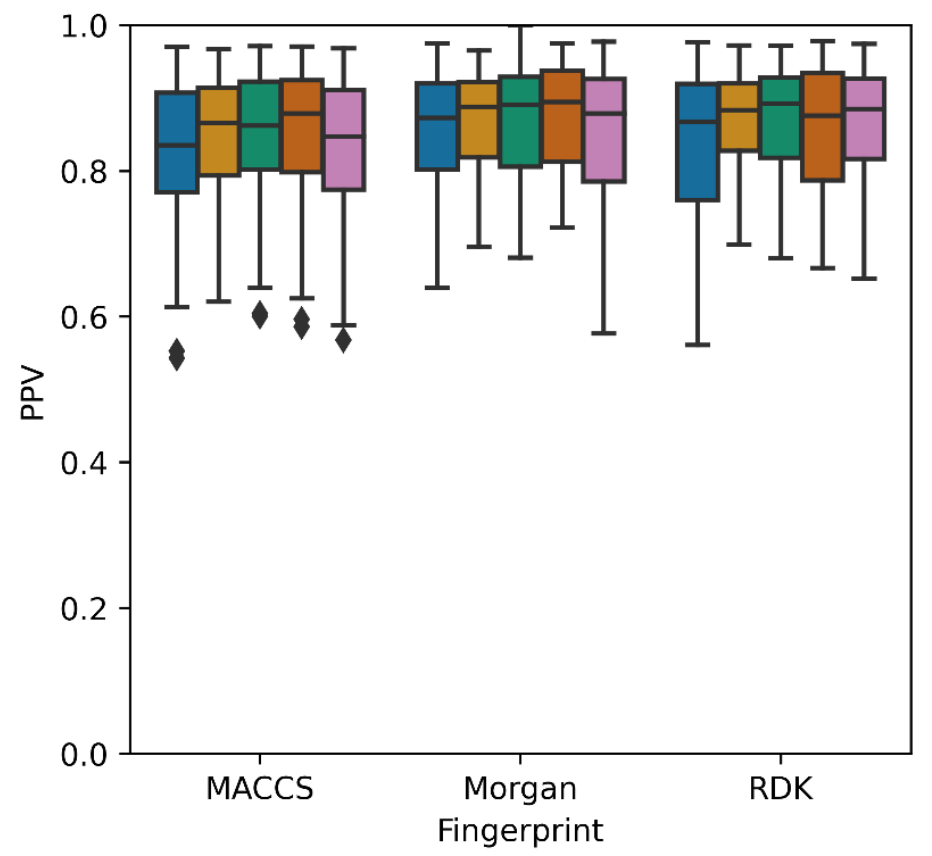

(c)

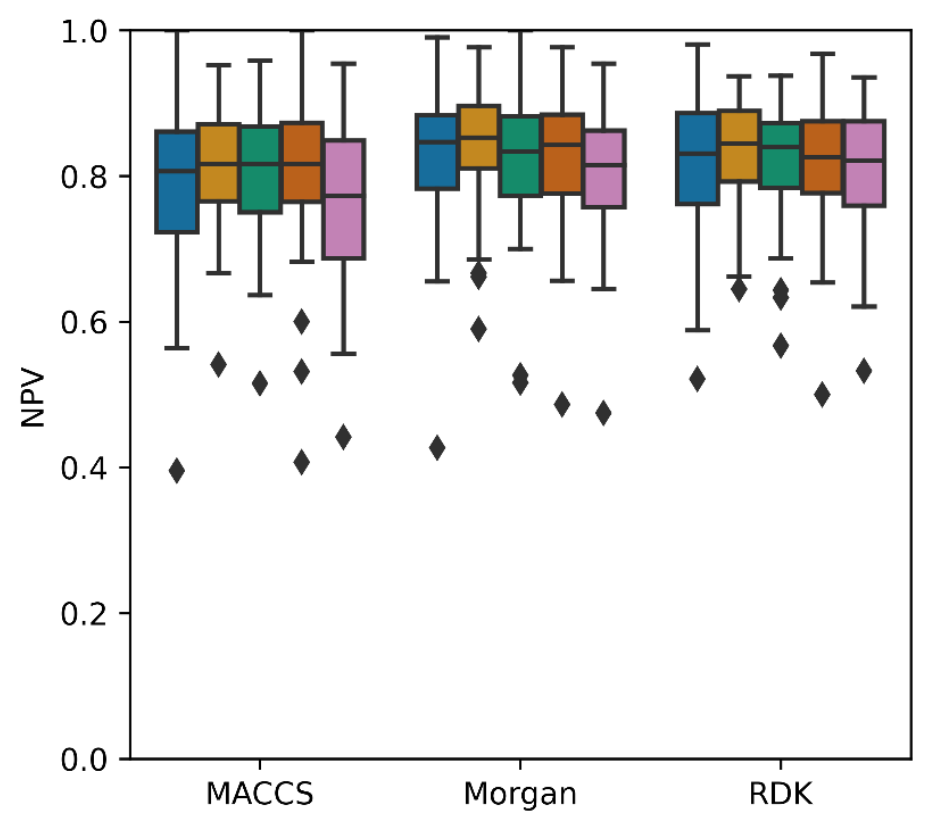

Fingerprint (b)

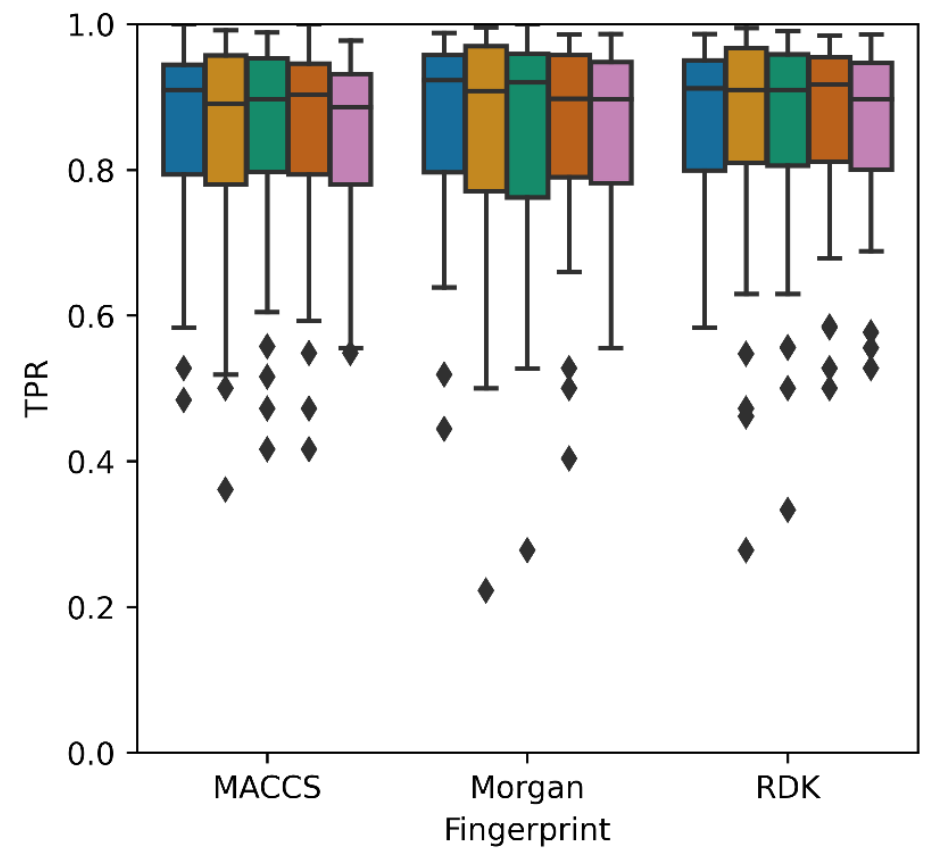

(d)

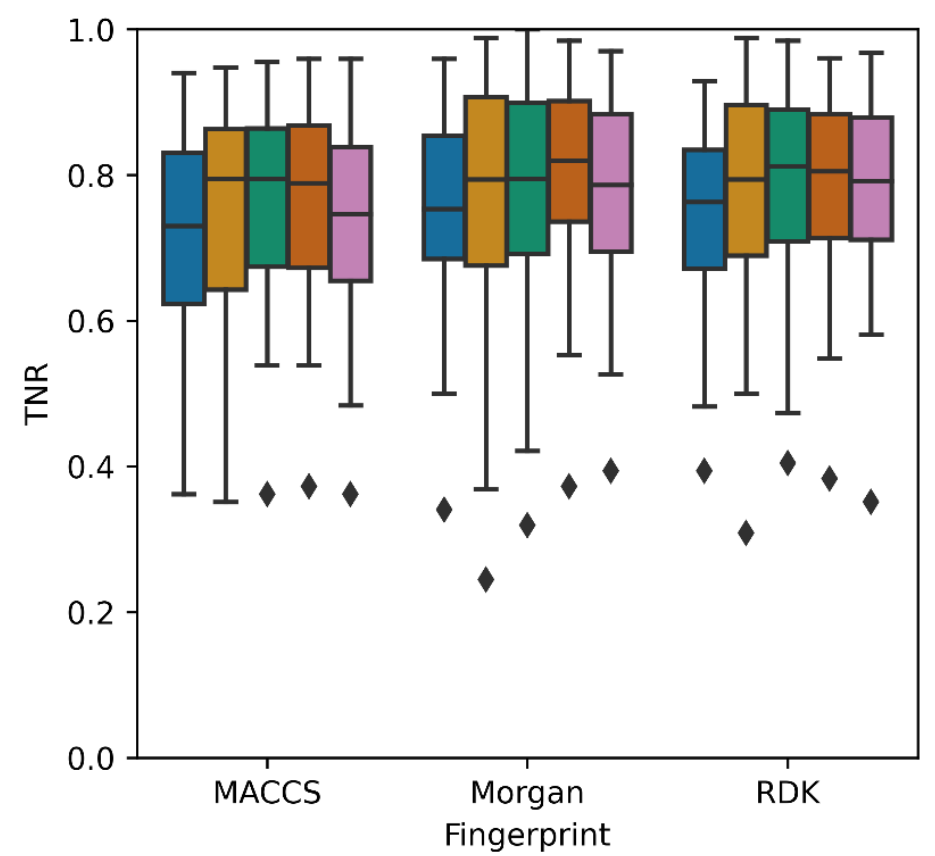

Fingerprint

$\square$ k-NN $\square$ RF $\square$ GBT $\square$ SVM $\square$ FFNN

Figure S1. Performance comparison of single target binary classifiers. Each boxplot contains the performance metrics for 55 different target-associated compound datasets. 


\begin{tabular}{|c|c|c|c|c|c|c|c|c|c|c|}
\hline Sample $1-$ & 197 & 24 & 25 & 31 & 32 & 20 & 22 & 28 & 26 & 29 \\
\hline Sample 2 - & 24 & 217 & 20 & 31 & 36 & 38 & 30 & 33 & 31 & 28 \\
\hline Sample 3 - & 25 & 20 & 184 & 28 & 24 & 22 & 16 & 19 & 23 & 23 \\
\hline Sample 4 - & 31 & 31 & 28 & 221 & 40 & 36 & 27 & 25 & 26 & 26 \\
\hline Sample 5 - & 32 & 36 & 24 & 40 & 211 & 27 & 19 & 23 & 33 & 25 \\
\hline Sample 6 - & 20 & 38 & 22 & 36 & 27 & 229 & 27 & 25 & 30 & 24 \\
\hline Sample 7 - & 22 & 30 & 16 & 27 & 19 & 27 & 206 & 26 & 30 & 38 \\
\hline Sample 8 - & 28 & 33 & 19 & 25 & 23 & 25 & 26 & 202 & 28 & 21 \\
\hline Sample 9 - & 26 & 31 & 23 & 26 & 33 & 30 & 30 & 28 & 224 & 26 \\
\hline \multirow[t]{5}{*}{ Sample 10 - } & 29 & 28 & 23 & 26 & 25 & 24 & 38 & 21 & 26 & 206 \\
\hline & 1 & I & 1 & 1 & 1 & 1 & 1 & 1 & 1 & \\
\hline & 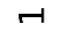 & $N$ & $m$ & $\nabla$ & เn & 6 & $N$ & $\infty$ & $\sigma$ & 0 \\
\hline & $\underline{\theta}$ & $\underline{\theta}$ & $\underline{\theta}$ & $\underline{\varphi}$ & $\underline{\omega}$ & $\underline{\theta}$ & $\underline{\theta}$ & $\underline{\omega}$ & $\underline{\theta}$ & r \\
\hline & $\begin{array}{l}\overline{\bar{\alpha}} \\
\bar{\varepsilon} \\
\text { ஸे }\end{array}$ & $\begin{array}{l}\overline{\mathrm{O}} \\
\bar{\varepsilon} \\
\bar{\sigma}\end{array}$ & $\begin{array}{l}\overline{\bar{\alpha}} \\
\bar{E} \\
\text { ஸ }\end{array}$ & $\begin{array}{l}\overline{ } \\
\text { 등 } \\
\text { ஸे }\end{array}$ & $\begin{array}{l}\overline{\mathrm{O}} \\
\bar{\varepsilon} \\
\text { ஸे }\end{array}$ & $\begin{array}{l}\overline{\mathrm{O}} \\
\bar{E} \\
\text { ஸे }\end{array}$ & $\begin{array}{l}\overline{\mathrm{O}} \\
\bar{\varepsilon} \\
\bar{\sigma}\end{array}$ & $\frac{\bar{\alpha}}{\bar{E}}$ & $\begin{array}{l}\overline{\mathrm{O}} \\
\bar{\varepsilon} \\
\bar{\sigma}\end{array}$ & $\frac{\frac{1}{0}}{\frac{2}{\sigma}}$ \\
\hline
\end{tabular}

Figure S2. Compounds overlap between samples employed in the Multi-Target Validation. 
Table S5. External compounds employed for retrospective target prediction.

\begin{tabular}{cr}
\hline Compound & SMILES \\
\hline $\mathbf{1 7}$ & $\mathrm{C}[\mathrm{C} @ \mathrm{H}](\mathrm{CN}[\mathrm{C} @ @ \mathrm{H}](\mathrm{C}(=\mathrm{O}) \mathrm{NC} 1=\mathrm{NC}=\mathrm{C}(\mathrm{C}=\mathrm{C} 1) \mathrm{C} 1=\mathrm{CN}(\mathrm{C}) \mathrm{N}=\mathrm{C} 1) \mathrm{C} 1=\mathrm{CC}=\mathrm{CC}=\mathrm{C} 1) \mathrm{C} 1=\mathrm{CC}=\mathrm{C}(\mathrm{C}=\mathrm{C} 1) \mathrm{C \# N}$ \\
$\mathbf{4 3 a}$ & $\mathrm{CC}(\mathrm{C}) \mathrm{OC} 1=\mathrm{CC}=\mathrm{C}(\mathrm{C}=\mathrm{C} 1 \mathrm{C} 1=\mathrm{CN}(\mathrm{C}) \mathrm{C}(=\mathrm{O}) \mathrm{C} 2=\mathrm{C} 1 \mathrm{C}=\mathrm{C}(\mathrm{N} 2) \mathrm{C}(=\mathrm{O}) \mathrm{NCCCCCC}(=\mathrm{O}) \mathrm{NO}) \mathrm{C}(\mathrm{C})(\mathrm{C}) \mathrm{O}$ \\
\hline
\end{tabular}

Table S6. Targets predicted by the consensus model for compound 17.

\begin{tabular}{ccc}
\hline Target & ChEMBL ID & Quartile \\
\hline CARM1 & CHEMBL5406 & Q3 \\
EZH2 & CHEMBL2189110 & Q3 \\
KDM1A & CHEMBL6136 & Q3 \\
ATM & CHEMBL3797 & Q4 \\
BRD2 & CHEMBL1293289 & Q4 \\
CDK1 & CHEMBL308 & Q4 \\
CDK2 & CHEMBL301 & Q4 \\
CDK7 & CHEMBL3055 & Q4 \\
CREBBP & CHEMBL5747 & Q4 \\
EP300 & CHEMBL3784 & Q4 \\
PARP1 & CHEMBL3105 & Q4 \\
PRMT3 & CHEMBL5891 & Q4
\end{tabular}

Table S7. Targets predicted by HitPickV2 for compound 17.

\begin{tabular}{cccc}
\hline Predicted/Known Target & Precision & Most Similar Compound & Tc \\
\hline FFAR2 & 26.7 & CHEMBL607315 & 0.301 \\
CDK5 & 26.3 & CHEMBL186934 & 0.302 \\
NPY5R & 23.4 & CHEMBL494593 & 0.3 \\
PIM1 & 22.8 & CHEMBL3318030 & 0.327 \\
CNR1 & 22.4 & CHEMBL572080 & 0.312 \\
AURKB & 21.4 & CHEMBL3318030 & 0.327 \\
CNR2 & 20.7 & CHEMBL572080 & 0.312 \\
MAPK1 & 20 & CHEMBL3318030 & 0.327 \\
NTRK1 & 14 & CHEMBL3318030 & 0.327 \\
YES1 & 14 & CHEMBL3318030 & 0.327 \\
\hline
\end{tabular}


Table S8. Targets predicted by PPB for compound 17.

\begin{tabular}{ccc}
\hline Rank & ChEMBL ID & Common name \\
\hline 1 & CHEMBL3717 & Hepatocyte growth factor receptor \\
2 & CHEMBL2815 & Nerve growth factor receptor Trk-A \\
3 & CHEMBL1844 & Macrophage colony stimulating factor receptor \\
4 & CHEMBL240 & HERG \\
5 & CHEMBL2971 & Tyrosine-protein kinase JAK2 \\
6 & CHEMBL4630 & Serine/threonine-protein kinase Chk1 \\
7 & CHEMBL2973 & Rho-associated protein kinase 2 \\
8 & CHEMBL4722 & Serine/threonine-protein kinase Aurora-A \\
9 & CHEMBL279 & Vascular endothelial growth factor receptor 2 \\
10 & CHEMBL2148 & Tyrosine-protein kinase JAK3 \\
11 & CHEMBL4898 & Neurotrophic tyrosine kinase receptor type 2 \\
12 & CHEMBL3231 & Rho-associated protein kinase 1 \\
13 & CHEMBL1862 & Tyrosine-protein kinase ABL \\
14 & CHEMBL325 & Histone deacetylase 1 \\
15 & CHEMBL1974 & Tyrosine-protein kinase receptor FLT3 \\
16 & CHEMBL262 & Glycogen synthase kinase-3 beta \\
17 & CHEMBL2599 & Tyrosine-protein kinase SYK \\
18 & CHEMBL4501 & Ribosomal protein S6 kinase 1 \\
19 & CHEMBL2185 & Serine/threonine-protein kinase Aurora-B \\
20 & CHEMBL3837 & Cathepsin L \\
\hline
\end{tabular}

Table S9. Targets predicted by SEA for compound 17.

\begin{tabular}{cccccc}
\hline Target ID & Affinity Threshold $(\mathbf{n M})$ & P-Value & Max Tc & Cut Sum & Z-Score \\
\hline BAZ2A_HUMAN & 5 & $4.93 \mathrm{E}-13$ & 0.3553 & 0.9867 & 21.6455 \\
BAZ2B_HUMAN & 5 & $9.53 \mathrm{E}-13$ & 0.3553 & 0.9867 & 21.1314 \\
CCNC_HUMAN & 5 & $4.61 \mathrm{E}-13$ & 0.3205 & 3.7686 & 21.6973 \\
CDKA1_HUMAN & 5 & $7.75 \mathrm{E}-06$ & 0.3023 & 0.3023 & 8.725 \\
CDK8_HUMAN & 5 & $8.41 \mathrm{E}-08$ & 0.3205 & 3.7686 & 12.252 \\
CECR2_HUMAN & 5 & $5.92 \mathrm{E}-06$ & 0.3553 & 0.3553 & 8.936 \\
DPP4_PIG & 5 & $5.14 \mathrm{E}-06$ & 0.2967 & 0.8614 & 9.0462 \\
F263_HUMAN & 5 & $5.82 \mathrm{E}-13$ & 0.3418 & 4.2543 & 21.5158 \\
GRIA3_HUMAN & 5 & $2.89 \mathrm{E}-07$ & 0.3571 & 0.3571 & 11.2887 \\
GRIA4_HUMAN & 5 & $6.37 \mathrm{E}-06$ & 0.3571 & 0.93 & 8.8779 \\
HXK4_HUMAN & 5 & $1.28 \mathrm{E}-08$ & 0.3511 & 5.1412 & 13.7212 \\
LUCI_AQULA & 5 & $1.02 \mathrm{E}-29$ & 0.3235 & 1.8545 & 51.6005 \\
MK01_HUMAN & 5 & $3.01 \mathrm{E}-12$ & 0.3875 & 16.1189 & 20.235 \\
MTP_HUMAN & 5 & $1.67 \mathrm{E}-11$ & 0.3077 & 2.3554 & 18.8993 \\
NTRK1_HUMAN & 5 & $1.35 \mathrm{E}-06$ & 0.3409 & 8.2579 & 10.0904 \\
O96214_PLAFA & 5 & $7.06 \mathrm{E}-25$ & 0.321 & 1.8428 & 42.9087 \\
PDPK1_HUMAN & 5 & $2.76 \mathrm{E}-06$ & 0.3222 & 3.5961 & 9.5307 \\
PORCN_HUMAN & 5 & $2.81 \mathrm{E}-17$ & 0.3924 & 1.738 & 29.2652 \\
PORCN_MOUSE & 5 & $1.30 \mathrm{E}-23$ & 0.3415 & 1.8349 & 40.6369 \\
\hline
\end{tabular}


Table S10. Top 45 targets predicted by SwissTargetPrediction for compound 17.

\begin{tabular}{|c|c|c|}
\hline Common name & ChEMBL ID & Probability* \\
\hline PLD1 & CHEMBL2536 & 0.120225751 \\
\hline PLD2 & CHEMBL2734 & 0.120225751 \\
\hline CAMK2D & CHEMBL2801 & 0.120225751 \\
\hline SCARB1 & CHEMBL1914272 & 0.120225751 \\
\hline SSTR2 & CHEMBL1804 & 0.120225751 \\
\hline CHRM2 & CHEMBL211 & 0.120225751 \\
\hline SLC6A2 & CHEMBL222 & 0.120225751 \\
\hline ACKR3 & CHEMBL2010631 & 0.120225751 \\
\hline PRKCl & CHEMBL2598 & 0.120225751 \\
\hline HTR2B & CHEMBL1833 & 0.120225751 \\
\hline CCR8 & CHEMBL4596 & 0.120225751 \\
\hline GNRHR & CHEMBL1855 & 0.120225751 \\
\hline ERBB2 & CHEMBL1824 & 0.120225751 \\
\hline MTNR1A & CHEMBL1945 & 0.120225751 \\
\hline MTNR1B & CHEMBL1946 & 0.120225751 \\
\hline TACR2 & CHEMBL2327 & 0.120225751 \\
\hline CASR & CHEMBL1878 & 0.120225751 \\
\hline PGGT1B FNTA & CHEMBL2095164 & 0.120225751 \\
\hline ERG & CHEMBL1293191 & 0.120225751 \\
\hline HTR4 & CHEMBL1875 & 0.120225751 \\
\hline HTR5A & CHEMBL3426 & 0.120225751 \\
\hline NEK1 & CHEMBL5855 & 0.120225751 \\
\hline LTA4H & CHEMBL4618 & 0.120225751 \\
\hline CNR1 & CHEMBL218 & 0.120225751 \\
\hline CHRNA4 CHRNB2 & CHEMBL1907589 & 0.120225751 \\
\hline CHRNA7 & CHEMBL2492 & 0.120225751 \\
\hline CCR5 & CHEMBL274 & 0.120225751 \\
\hline HDAC5 & CHEMBL2563 & 0.120225751 \\
\hline EBP & CHEMBL4931 & 0.120225751 \\
\hline TK1 & CHEMBL2883 & 0.120225751 \\
\hline CYP26A1 & CHEMBL5141 & 0.120225751 \\
\hline TRHR & CHEMBL1810 & 0.120225751 \\
\hline SLC18A2 & CHEMBL1893 & 0.120225751 \\
\hline $\mathrm{KIF} 11$ & CHEMBL4581 & 0.120225751 \\
\hline CACNA2D1 & CHEMBL1919 & 0.120225751 \\
\hline DBF4 CDC7 & CHEMBL2111377 & 0.120225751 \\
\hline CDC7 & CHEMBL5443 & 0.120225751 \\
\hline HSP90AB1 & CHEMBL4303 & 0.120225751 \\
\hline PTGER2 & CHEMBL1881 & 0.120225751 \\
\hline KDM5C & CHEMBL2163176 & 0.120225751 \\
\hline CTSK & CHEMBL268 & 0.120225751 \\
\hline KDM4B & CHEMBL3313832 & 0.120225751 \\
\hline KDM5B & CHEMBL3774295 & 0.120225751 \\
\hline LYN & CHEMBL3905 & 0.120225751 \\
\hline KDM4A & CHEMBL5896 & 0.120225751 \\
\hline
\end{tabular}


Table S11. Targets predicted by the consensus model for compound 43a.

\begin{tabular}{ccc}
\hline ChEMBL ID & Gene & Quartile \\
\hline CHEMBL325 & HDAC1 & Q2 \\
CHEMBL1829 & HDAC3 & Q2 \\
CHEMBL2563 & HDAC5 & Q2 \\
CHEMBL1865 & HDAC6 & Q2 \\
CHEMBL2716 & HDAC7 & Q2 \\
CHEMBL3192 & HDAC8 & Q2 \\
CHEMBL1163125 & BRD4 & Q3 \\
CHEMBL2189110 & EZH2 & Q3 \\
CHEMBL1937 & HDAC2 & Q3 \\
CHEMBL3524 & HDAC4 & Q3 \\
CHEMBL3797 & ATM & Q4 \\
CHEMBL1293289 & BRD2 & Q4 \\
CHEMBL5747 & CREBBP & Q4 \\
CHEMBL2971 & JAK2 & Q4 \\
CHEMBL6175 & KDM4C & Q4 \\
CHEMBL2424504 & KDM5A & Q4 \\
CHEMBL3105 & PARP1 & Q4 \\
CHEMBL3045 & PRKCB & Q4
\end{tabular}

Table S12. Targets predicted by HitPickV2 for compound 43a.

\begin{tabular}{cccc}
\hline Predicted/Known Target & Precision & Most Similar Compound & Tc \\
\hline HDAC6 & 38.2 & CHEMBL3309284 & 0.336 \\
HDAC3 & 30.3 & CHEMBL3309284 & 0.336 \\
HDAC10 & 26.7 & CHEMBL2364628 & 0.324 \\
HDAC5 & 26.3 & CHEMBL2364628 & 0.324 \\
HDAC7 & 23.4 & CHEMBL2364628 & 0.324 \\
HDAC2 & 23.2 & CHEMBL3309284 & 0.336 \\
HDAC8 & 22.8 & CHEMBL3309284 & 0.336 \\
HDAC9 & 21.4 & CHEMBL3309284 & 0.336 \\
HDAC1 & $0.0^{*}$ & CHEMBL3309284 & 0.336 \\
HDAC4 & $15.7^{*}$ & CHEMBL3309284 & 0.336 \\
\hline
\end{tabular}


Table S13. Targets predicted by PPB for compound 43a.

\begin{tabular}{ccc}
\hline Rank & ChEMBL ID & Common name \\
\hline 1 & CHEMBL1865 & Histone deacetylase 6 \\
2 & CHEMBL3192 & Histone deacetylase 8 \\
3 & CHEMBL235 & Peroxisome proliferator-activated receptor gamma \\
4 & CHEMBL2599 & Tyrosine-protein kinase SYK \\
5 & CHEMBL325 & Histone deacetylase 1 \\
6 & CHEMBL1937 & Histone deacetylase 2 \\
7 & CHEMBL332 & Matrix metalloproteinase-1 \\
8 & CHEMBL253 & Cannabinoid CB2 receptor \\
9 & CHEMBL3524 & Histone deacetylase 4 \\
10 & CHEMBL217 & Dopamine D2 receptor \\
11 & CHEMBL283 & Matrix metalloproteinase 3 \\
12 & CHEMBL5103 & Histone deacetylase 10 \\
13 & CHEMBL333 & Matrix metalloproteinase-2 \\
14 & CHEMBL2563 & Histone deacetylase 5 \\
15 & CHEMBL1829 & Histone deacetylase 3 \\
16 & CHEMBL4145 & Histone deacetylase 9 \\
17 & CHEMBL321 & Matrix metalloproteinase 9 \\
18 & CHEMBL1946 & Melatonin receptor 1B \\
19 & CHEMBL2716 & Histone deacetylase 7 \\
20 & CHEMBL4588 & Matrix metalloproteinase 8 \\
\hline & & \\
& &
\end{tabular}

Table S14. Targets predicted by SEA for compound 43a.

\begin{tabular}{cccccc}
\hline Target ID & Affinity Threshold (nM) & P-Value & Max Tc & Cut Sum & Z-Score \\
\hline A5H660_SCHMA & 5 & $6.25 \mathrm{E}-06$ & 0.3421 & 0.6261 & 8.8934 \\
BRD4_HUMAN & 5 & $1.87 \mathrm{E}-52$ & 0.4239 & 51.3602 & 92.4181 \\
ENPP2_MOUSE & 5 & $8.32 \mathrm{E}-13$ & 0.2824 & 0.2824 & 21.2374 \\
HDA11_HUMAN & 5 & $5.30 \mathrm{E}-06$ & 0.3229 & 1.2404 & 9.022 \\
HDA10_HUMAN & 5 & $2.61 \mathrm{E}-17$ & 0.3333 & 4.9288 & 29.3212 \\
HDAC1_HUMAN & 5 & $1.06 \mathrm{E}-20$ & 0.3875 & 33.7458 & 35.4096 \\
HDAC1_RAT & 5 & $2.71 \mathrm{E}-06$ & 0.3421 & 0.3421 & 9.5448 \\
HDAC1_MOUSE & 5 & $7.23 \mathrm{E}-06$ & 0.3182 & 0.6103 & 8.7797 \\
HDAC2_HUMAN & 5 & $2.80 \mathrm{E}-12$ & 0.3875 & 8.2769 & 20.2922 \\
HDAC4_HUMAN & 5 & $8.02 \mathrm{E}-08$ & 0.3875 & 3.6816 & 12.2894 \\
HDAC5_HUMAN & 5 & $7.57 \mathrm{E}-09$ & 0.3229 & 1.5427 & 14.1299 \\
HDAC3_HUMAN & 5 & $3.01 \mathrm{E}-26$ & 0.3875 & 21.6303 & 45.3695 \\
HDAC6_HUMAN & 5 & $1.44 \mathrm{E}-48$ & 0.4079 & 54.6742 & 85.4422 \\
HDAC9_HUMAN & 5 & $6.10 \mathrm{E}-20$ & 0.3875 & 3.6816 & 34.0466 \\
HDAC8_HUMAN & 5 & $2.64 \mathrm{E}-19$ & 0.3875 & 14.5609 & 32.904 \\
NCOR2_HUMAN & 5 & $1.24 \mathrm{E}-07$ & 0.3229 & 1.2286 & 11.9494 \\
Q94F81_MAIZE & 5 & $6.83 \mathrm{E}-29$ & 0.3784 & 5.6794 & 50.1164 \\
A5H660_SCHMA & 5 & $6.25 \mathrm{E}-06$ & 0.3421 & 0.6261 & 8.8934 \\
BRD4_HUMAN & 5 & $1.87 \mathrm{E}-52$ & 0.4239 & 51.3602 & 92.4181 \\
\hline
\end{tabular}


Table S15. Top 45 targets predicted by SwissTargetPrediction for compound 43a.

\begin{tabular}{|c|c|c|}
\hline Common name & ChEMBL ID & Probability* \\
\hline PIK3CA PIK3R1 & CHEMBL2111367 & 0.12190626 \\
\hline ESR2 & CHEMBL242 & 0.12190626 \\
\hline S1PR1 & CHEMBL4333 & 0.12190626 \\
\hline ESR1 & CHEMBL206 & 0.12190626 \\
\hline NCOR2 HDAC3 & CHEMBL2111363 & 0.12190626 \\
\hline PIK3CD PIK3R1 & CHEMBL2111432 & 0.12190626 \\
\hline PIK3CB & CHEMBL3145 & 0.12190626 \\
\hline PIK3CG & CHEMBL3267 & 0.12190626 \\
\hline FLT1 & CHEMBL1868 & 0.12190626 \\
\hline ESRRA & CHEMBL3429 & 0.12190626 \\
\hline ESRRB & CHEMBL3751 & 0.12190626 \\
\hline FLT3 & CHEMBL1974 & 0 \\
\hline PTPN1 & CHEMBL335 & 0 \\
\hline S1PR3 & CHEMBL3892 & 0 \\
\hline S1PR5 & CHEMBL2274 & 0 \\
\hline S1PR4 & CHEMBL3230 & 0 \\
\hline SLC5A2 & CHEMBL3884 & 0 \\
\hline SLC5A1 & CHEMBL4979 & 0 \\
\hline VCP & CHEMBL1075145 & 0 \\
\hline MMP1 & CHEMBL332 & 0 \\
\hline FYN & CHEMBL1841 & 0 \\
\hline ABL1 & CHEMBL1862 & 0 \\
\hline PDGFRB & CHEMBL1913 & 0 \\
\hline CCNT1 & CHEMBL2108 & 0 \\
\hline ALK & CHEMBL4247 & 0 \\
\hline ERBB2 & CHEMBL1824 & 0 \\
\hline EGFR & CHEMBL203 & 0 \\
\hline ADRB2 & CHEMBL210 & 0 \\
\hline TACR2 & CHEMBL2327 & 0 \\
\hline PPIA & CHEMBL1949 & 0 \\
\hline ADK & CHEMBL3589 & 0 \\
\hline DUT & CHEMBL5203 & 0 \\
\hline SLC2A1 & CHEMBL2535 & 0 \\
\hline INSR & CHEMBL1981 & 0 \\
\hline ITK & CHEMBL2959 & 0 \\
\hline PIM3 & CHEMBL5407 & 0 \\
\hline TYMS & CHEMBL1952 & 0 \\
\hline EGLN2 & CHEMBL3028 & 0 \\
\hline TACR3 & CHEMBL4429 & 0 \\
\hline EGLN3 & CHEMBL5705 & 0 \\
\hline EGLN1 & CHEMBL5697 & 0 \\
\hline HDAC6 & CHEMBL1865 & 0 \\
\hline HDAC8 & CHEMBL3192 & 0 \\
\hline HDAC1 & CHEMBL325 & 0 \\
\hline CHRM1 & CHEMBL216 & 0 \\
\hline
\end{tabular}

